\title{
Uso de sensores industriales en la preparación de alimentos
}

Use of industrial sensors in food preparation

Recibido: abril de 2016 | Revisado: mayo de 2016 | Aceptado: junio de 2016

Milagros Quispe $^{1}$
Jorge Calderón $^{2}$
1 Área de Diseño Gráfico y Multimedia, Universidad de San Martín de Porres, Av. La Fontana 1250. La Molina. Perú. aquisper@usmp.pe

2 Área de Control y Automatización, Universidad de San MArtín de Porres, Av. La Fontana 1250. La Molina. Perú. jcalderonc@usmp.pe

\begin{abstract}
In the current study, we assess the need of using thermal sensors in food preparation machines, taking as an example the development of frejol colado, sweet typical of our country, whose preparation requires constant and continuous movement when mixing ingredients, exposing people who are dedicated to this task to high volumes of heat, which lead in time leads to health issues. Frejol colado is known as a specialty of unique taste. This special flavor has been spreading and gaining great acceptance by consumers. Nowadays, to ensure the safety and quality of the product, it is necessary that the cooking process is carried out using good manufacturing practices and careful processing, preservation and presentation in order to meet at minimum, the needs of potential customers by offering a quality product. The effects of overlapping heat while cooking can be diverse and not only immediate but over time, and can cause serious health problems of people who are dedicated to the task of cooking. First, by using tools that transmit heat by themselves, as well as having both hands and arms are exposed to steam heat of a kitchen. They may also present alterations in lung health, since studies indicate that the use of a gas oven when cooking can increase asthma symptoms.
\end{abstract}

Keywords: temperature sensors, safety, quality, conservation, overlap

\section{Resumen}

En el presente estudio, se hace una evaluación de la necesidad de utilizar sensores térmicos en máquinas de preparación de alimentos, tomando como ejemplo la elaboración del frejol colado, dulce típico de nuestro país, cuya preparación requiere de un movimiento constante y continuo al momento de mezclar los ingredientes, y exponer a las personas que se dedican a esta tarea a altos volúmenes de calor, lo que conlleva a problemas posteriores en su salud. El frejol colado es conocido como una especialidad de sabor único. Este especial sabor fue difundiéndose y obteniendo gran aceptación por parte de sus consumidores. Actualmente, para garantizar la inocuidad y calidad del producto es necesario que la elaboración se realice aplicando las buenas prácticas de fabricación y con los cuidados de elaboración, conservación y presentación necesarios para cumplir, como mínimo, con las necesidades de los clientes potenciales ofreciendo un producto de calidad. Los efectos de la superposición al calor al cocinar pueden ser diversos y no solo inmediatos, sino con el tiempo pueden ocasionar problemas graves en la salud de las personas que se dedican a cocinar. Primero al utilizar utensilios que de por sí transmiten calor, así como el que las manos y brazos se expongan al calor del vapor de una cocina. También se pueden presentar efectos en la salud pulmonar, ya que estudios indican que la utilización de una cocina de gas al cocinar puede aumentar los síntomas de asma.

Palabras clave: sensores térmicos, inocuidad, calidad, conservación, superposición 


\section{Introducción}

Hoy en día, la automatización cumple un papel muy importante en la industria, la elaboración de sistemas de fabricación diseñados con el fin de usar la capacidad de las máquinas para llevar a cabo determinadas tareas, anteriormente efectuadas por seres humanos, y para controlar la secuencia de las operaciones sin intervención humana. En la actualidad, estos sistemas se utilizan para efectuar diversas tareas con más rapidez o mejor de lo que podría hacerlo un ser humano.

Precisamente, este desarrollo de novedosos prototipos, máquinas o materiales permite una mayor rapidez a aquellas actividades en las cuales se emplea un mayor tiempo. Asimismo, para que se ofrezca seguridad a las personas que realizan actividades que pueden poner en riesgo su salud, como es el estar expuestos al calor de una cocina o materiales calientes durante muchas horas al día, que pueden originar quemaduras.

Según la Organización Mundial de la Salud, cada año, las quemaduras ocasionan, aproximadamente, 265000 muertes al año, que en su gran mayoría tienen lugar en los países de ingreso bajo y mediano. Las lesiones por quemadura no fatales son una de las principales causas de morbilidad. Las quemaduras se producen mayormente en el ámbito doméstico y laboral. Dentro de las alternativas de mejora, surge la idea de una máquina automatizada que permita desarrollar la actividad de la producción de frejol colado. Actualmente, para elaborar este alimento se requiere un movimiento constante con una cuchara de madera, aproximadamente, 30 minutos hasta que tome el color y punto necesarios.

Una amplia variedad de sensores se pueden emplear, en el diseño de sistemas instrumentales, especialmente las lenguas electrónicas, sobre todo tratándose de alimentos, que ofrecen numerosas aplicaciones prácticas (Tudor, Mar Kovic, Kalit, Vahčić \& Karranek, 2014).
Una máquina automatizada de frejol colado permitiría realizar el mismo postre de una manera sencilla y sin que una persona se exponga a diversos accidentes que pueden afectar su salud en el corto, mediano y largo plazo.

\section{Sensores térmicos}

Los sensores de interés, para la manufactura, se pueden clasificar en sensores mecánicos, eléctricos, magnéticos y térmicos. También se tienen los acústicos, ultrasónicos, químicos, ópticos, de radiación, láser y de fibra óptica (Kalpakjian \& Schmid,, 2002) que se utilizan para medir temperatura, flujo, conductividad y calor específico.

La medida de temperatura en los fluidos es mucho más compleja, y en especial, depende de la viscosidad, conductividad térmica y velocidad del fluido. Es necesario, a su vez, tener en cuenta que el frotamiento entre el fluido y el sensor produce un calentamiento del sensor, por lo que su temperatura se elevaría (Consejo Superior de Investigaciones Científicas [CSIC], 1987).

Los sensores de gas se utilizan para detectar diferencias en los alimentos; sin embargo, estas aplicaciones están limitadas para el seguimiento de los alimentos, ya que realizan la identificación estricta, que es poco realista, en un entorno de cocina dinámico, debido al aislamiento del aroma que típicamente requieren, así como el aumento de la dificultad de identificar una sustancia en especial (Hirano, 2013).

Es importante analizar los tipos de sensores que debemos utilizar según lo que se requiera como resultado y el que mejor información pueda proporcionar, ya que de ello dependerá la calidad del producto que se elabore en la industria alimenticia.

Las técnicas clásicas de análisis descriptivo, a saber, el perfil de sabor, análisis descriptivo cuantitativo y perfil de la textura, son 
principalmente útiles para la caracterización sensorial de los productos alimenticios. Estos métodos sensoriales necesitan un grupo de asesores bien entrenados y varios atributos establecidos con el fin de proporcionar resultados fiables; requisitos que exigen mucho tiempo y, en algunos casos, podrían causar graves problemas en algunas industrias y laboratorios (Romani, Balestra, Angioloni, Rocculi \& Dalla, 2011).

Las narices electrónicas emplean diferentes tipos de sensores de gas electrónicos que tienen especificidad parcial y una técnicas de reconocimiento de patrones apropiados capaces de reconocer los olores simples y complejos (Zahora, Khan, Srivasava \& Hunderwale, 2013).

Probablemente, sea la temperatura el parámetro físico más común que se mide en una aplicación electrónica, incluso en muchos casos, en que el parámetro de interés no es la temperatura, esta se ha de medir para incluir indirectamente su efecto en la medida deseada (Alzate, Montes \& Silva, 2007).

En el mercado, tenemos los siguientes sensores de temperatura, más utilizados en las máquinas procesadoras de alimentos: a. Sensor de termorresistencia. También conocido como RTD, por sus siglas en inglés, es un detector de temperatura por resistencia, es decir, un sensor de temperatura que detecta la variación de la resistencia de un determinado conductor en función de la temperatura del ambiente.

b. Sensor bimetálico. Con contactos eléctricos los que cierran y abren los circuitos eléctricos en función de la posición de la aguja de los instrumentos de medición. Es una tira compuesta por dos chapas de metal de diferentes coeficientes de dilatación ("bimetal"), laminadas entre sí, en forma inseparable, se deforma a consecuencia de un cambio de temperatura.

c. La termocupla o termopar. Es la unión de dos alambres, según sea el caso, el hierro y el imbar (Tipo J) y al quemarse se abre y debe ser reemplazado, las termocuplas más usadas son el tipo J y el tipo K (Chromel y Alumel). En la Figura 1, podemos ver una comparación de los sensores más utilizados en la actualidad.

\begin{tabular}{|c|c|c|c|}
\hline & \multicolumn{3}{|c|}{ Sensores } \\
\hline & Termocupla & Termoresistencias & Bimetálico \\
\hline Rango & -200 a $2800^{\circ} \mathrm{C}$ & -250 a $850^{\circ} \mathrm{C}$ & -195 a $760^{\circ} \mathrm{C}$ \\
\hline Tipos & $\mathrm{J}, \mathrm{K}, \mathrm{S}, \mathrm{E}$ & RTD, PT 100 & $\begin{array}{l}\text { Relé de retardo, } \\
\text { contacto para PLC }\end{array}$ \\
\hline Costo & S/. $40.00 \times$ metro & S/. 250 & $\mathrm{~S} / .245$ \\
\hline Estabilidad & Baja & Alta & Media \\
\hline Exactitud & Media & Alta & Alta \\
\hline \multirow[t]{3}{*}{ Ventajas } & Económico & Más estable & Fácil de leer \\
\hline & Autoalimentado & Más preciso & $\begin{array}{l}\text { Menos sujeto a } \\
\text { quiebre que los de } \\
\text { vidrio }\end{array}$ \\
\hline & \begin{tabular}{|l} 
Amplia gama de \\
Temperaturas
\end{tabular} & $\begin{array}{l}\text { Más lineal que los } \\
\text { Termopares }\end{array}$ & Fácil de leer \\
\hline \multirow[t]{3}{*}{ Desventajas } & No lineal & Caro & $\begin{array}{l}\text { No tiene buena } \\
\text { precisión }\end{array}$ \\
\hline & Menos estable & $\begin{array}{l}\text { Precisa fuente de } \\
\text { alimentación }\end{array}$ & $\begin{array}{l}\text { Igual a los sensores de } \\
\text { tubo de vidrio }\end{array}$ \\
\hline & Menos sensible & Lento & \\
\hline
\end{tabular}

Fuente: Elaboración propia.

Figura 1. Tabla comparativa de sensores de temperatura eléctrica. 
En la Figura 1, podemos ver la tabla comparativa, en la cual se muestra el análisis que se hizo de los sensores más convenientes para la construcción de la máquina de producción de frejol colado, es el sensor PT 100 ya que es más estable y exacto en mediciones, según se puede ver en el mercado.

La tecnología ha ayudado, en la cocina, durante mucho tiempo, los termómetros para medir el nivel de cocción y las computadoras para medir el grado de cocción que son muy utilizados de diversas formas. No todos los escenarios de cocina son modificables para el uso de la visión o de los termómetros porque la comida puede ser obstruida visualmente, y termómetros de inserción pueden ser un inconveniente (Hirano, Brubaker, Patterson \& Hayes, 2013).

\section{Factores de riesgo y efectos sobre la piel por exposición al calor}

Al cocinar se debe tener cuidado porque se puede sufrir quemaduras cuyas causas se deben principalmente a:

a Fuego o llama, estar en contacto directo con el fuego o llama de los equipos en los cuales se cocina.

b Escaldado a raíz de vapor o líquidos calientes, al estar en contacto con el vapor, ya que muchos alimentos generan bastante vapor al cocinar y algunos alimentos requieren el movimiento constante por parte de la persona. Al pasar un tiempo, en la cocina, pueden sufrir quemaduras por el vapor, las mismas que son extremadamente dolorosas. Dichas quemaduras son clasificadas como de segundo grado debido al enrojecimiento, hinchazón o las ampollas que se generan por este contacto.

También podemos ver efectos futuros en la salud de las personas que cocinan, al estar continuamente en contacto del calor como son las enfermedades reumáticas y artríticas y también efectos bronquiales que pueden generar asma.

\section{Estado de la industrialización en la preparación de alimentos}

Hace muchos ańos atrás, nuestros ancestros llevaban sus alimentos (papa y otros tubérculos) a las zonas andinas más altas, estos se congelaban en las noches y se secaban desde ese estado durante el día, logrando la conservación de ellos. Hoy en día, la industria realiza este proceso, emulando mediante la tecnología, técnicas industriales de preservación de los alimentos (frutas, verduras, etc.), logrando salvaguardar hasta dos años a más, diversos productos, que mantengan todas sus propiedades organolépticas.

Para la preparación de alimentación se usan diversos ingredientes y materiales que permiten llegar al punto correcto de cocción. Se pueden utilizar cocinas de gas, cocinas eléctricas, horno microondas, cocina de inducción y vitrocerámica y, en muchas zonas de nuestro país, aún se cocina con leña y carbón.

La cocina de gas consume menos energía, es más rápida que la cocina vitrocerámica $\mathrm{y}$, además, admite todo tipo de recipientes. Son las más utilizadas por profesionales y por quienes apuestan por la cocina tradicional.

La cocina eléctrica tiene un costo menor, pero consumen mucha energía y son difíciles de limpiar. Las cocinas con placas vitrocerámicas son cómodas para limpiar, permiten todo tipo de recipientes, pero necesitan mucho más tiempo para calentarse que el gas y la inducción y, por tanto, consume más energía.

La utilización de una cocina de gas para cocinar puede aumentar los síntomas de asma, en el estudio elaborado en el 
Centro de Investigación en Epidemiología Ambiental de Barcelona, se obtuvo un aumento de la reactividad bronquial, una característica del asma relacionada con la contracción de las vías respiratorias pequeńas (Amaral, et. al., 2014).

El cocinar con leña o carbón puede producir efectos nocivos para la salud de las personas y esto se ha comprobado, en diversos estudios a nivel mundial. En la Figura 2, se muestran las emisiones de los principales contaminantes tóxicos, derivados de la leña por cada comida preparada en las cocinillas típicas de la India, en comparación con el combustible limpio más fácilmente disponible, el gas de petróleo licuado (GPL).

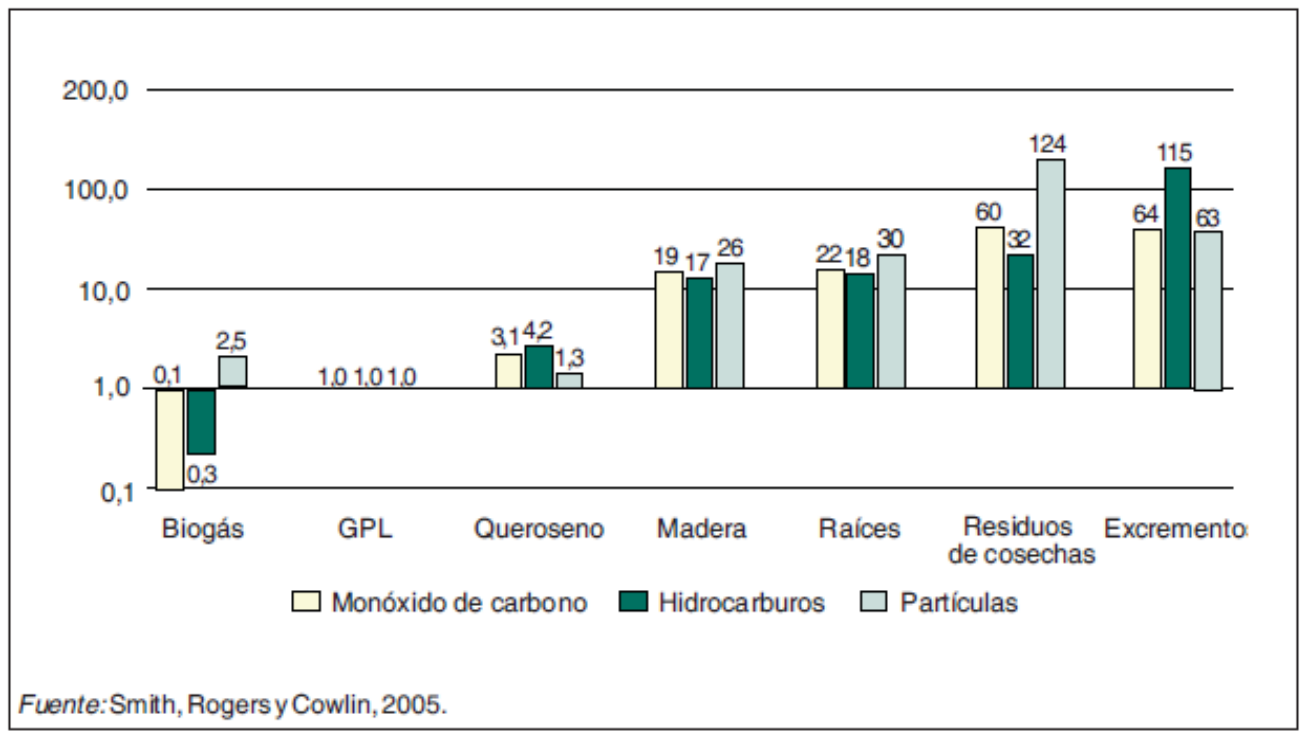

Figura 2. Escala de la energía: emisiones contaminantes por comida según el combustible utilizado, en relación con el GPL (1,0 en la escala) (medición hecha en la India) (Smith, 2006).

En los hogares pobres de los países en desarrollo, la leña, el carbón vegetal y otros combustibles sólidos (principalmente residuos agrícolas y carbón) se queman, a menudo, en fogones abiertos o estufas de mal funcionamiento. La combustión incompleta libera pequeñas partículas de otros componentes nocivos para la salud (Smith, 2006).

En las nuevas directrices de la Organización Mundial de la Salud, se recomienda no utilizar el carbón que es tratado como combustible doméstico. El carbón contiene elementos tóxicos como arsénico, plomo y mercurio. La combustión incompleta de carbón, en fogones y estufas poco eficientes, puede provocar enfermedades graves y muerte prematura.

\section{Objetivos del estudio}

El objetivo de este estudio fue analizar el impacto de la elaboración de una máquina procesadora de alimentos en la producción y elaboración del frejol colado y como su uso tendrá un impacto positivo en la salud y seguridad de las personas que cocinan este postre.

El análisis sensorial se ha utilizado durante mucho tiempo para la evaluación del olor. Sin embargo, las respuestas del panel sensorial tienen problemas potenciales (reproducibilidad, dificultades o la expresión y estabilidad) (Korel \& Balaban, 2003).

Evaluar el impacto, en la salud, que puede ocasionar el estar en contacto con equipos y materiales que generan calor en el momento de cocinar. 
Es importante calcular las ventajas en el uso de máquinas automatizadas, para la elaboración de alimentos.

Como en todos los procesos de la industria, existen riesgos al estar involucrados en parámetros del ojo humano. Al salir de control de parámetros normales de proceso, se incrementa el riesgo y, por lo tanto, las probabilidades de registrar pérdidas. Por eso es primordial mantener confiables los sistemas de control de proceso (Quispe \& Calderón, 2014).

Por tanto, elaborar una máquina con las especificaciones necesarias para la producción del frejol colado y que debe cumplir con los lineamientos de calidad del producto.

Muchos compuestos volátiles son responsables del aroma de los alimentos, y juegan un papel importante en el sabor. Estos volátiles contribuyen en la naturaleza de la comida, y preferencias de los consumidores. También pueden ser responsables de la ocurrencia de malos sabores y contaminaciones, debido a los cambios microbianos, bioquímica, química y, o contaminación (Hodgins, 1997).

\section{Materiales y métodos}

\section{a. Método de preparación manual del frejol colado}

Actualmente, para la elaboración del frejol colado se remojan los frejoles desde el día anterior. Al día siguiente se cocinan con agua hirviendo hasta que estén bien cocidos, se pelan, licuan y luego se pasa el producto por un colador. En una olla, se colocan los frejoles licuados, la leche, el azúcar, la canela, el clavo de olor y se cocina removiendo constantemente hasta que esté bien espeso, de color marrón y se vea el fondo de la olla. Al finalizar se retira del fuego y se deja enfriar.
Todo este proceso requiere de un tiempo y mucha paciencia para que el producto final pueda ser de calidad y con el sabor deseado. Como hemos podido apreciar, en la forma de cocinarlo, el contacto con el calor es constante y se requiere de mucho cuidado por parte de los que cocinan, ya que por un pequeño descuido se puede perder el producto y pueden ocurrir accidentes de quemaduras.

\section{b. Preparación del prototipo de la má- quina de frejol colado}

Máquina elaborada con acero inoxidable AISI 304, con cuatro soportes para la parte de la olla y además, dos soportes para el motor. La base de la olla es de forma circular para obtener un buen movimiento de giro de las paletas. Tiene una entrada de gas manual para poder regular la presión del fuego. El recipiente para la mezcla está hecho de acero inoxidable AISI 304.

En la parte eléctrica y electrónica de la máquina, se utilizaron los siguientes componentes:

- Relés

- Sensor de color

- Sensor de temperatura

- Llave termomagnética

- Display de temperatura

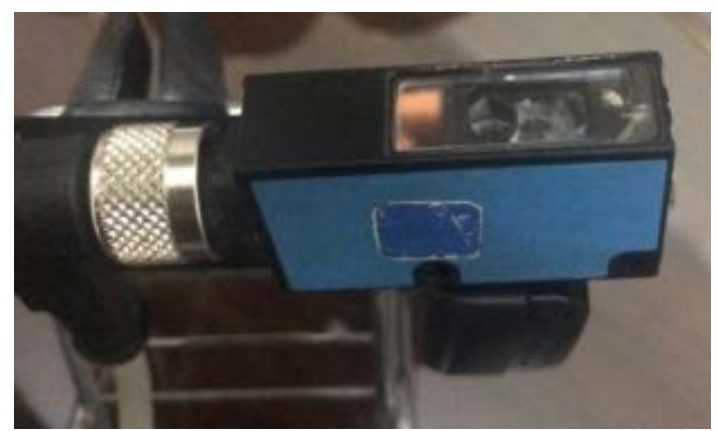

Figura 3. Sensor Intelligence SICK, fabricación Alemania.

Fuente: Elaboración propia. 


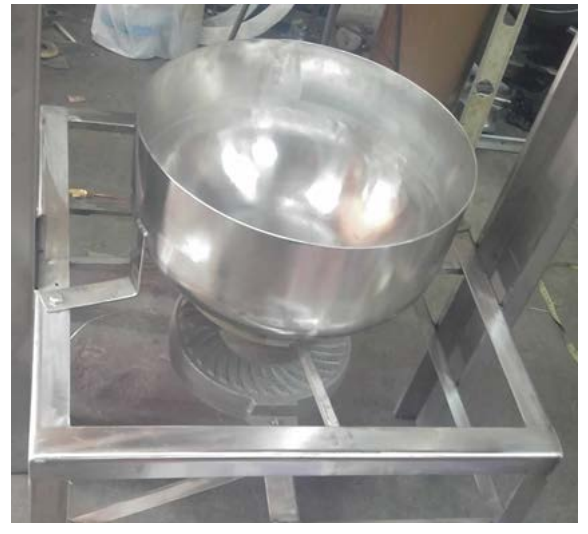

Figura 4. Soporte de olla.

Fuente: Elaboración propia.

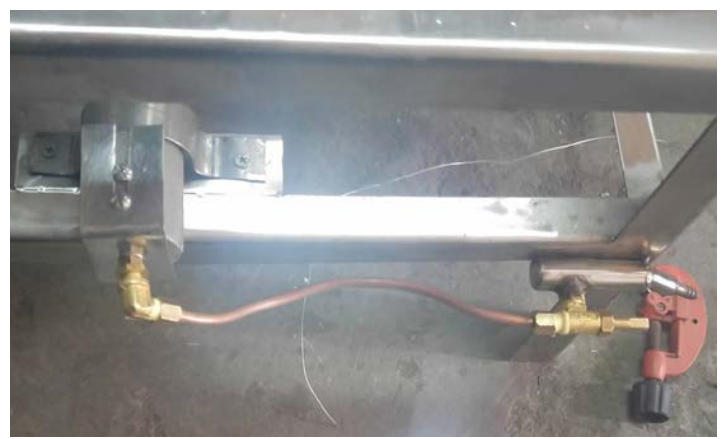

Figura 5. Electroválvula de control de gas Fuente: Elaboración propia.

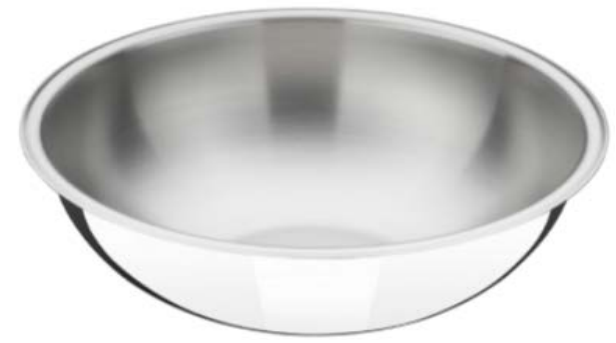

Figura 6. Recipiente para mezcla Fuente: Elaboración propia.

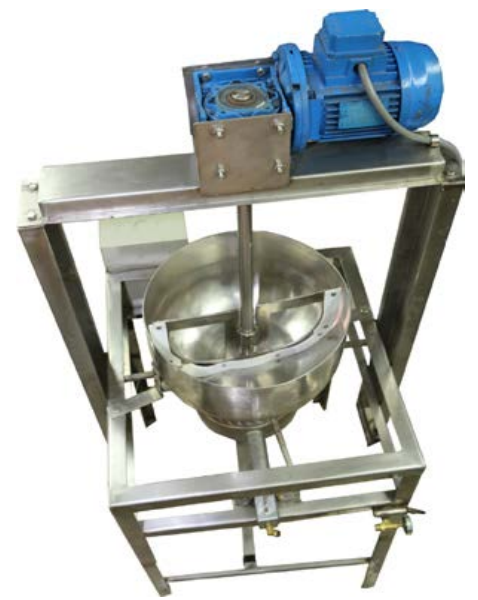

Figura 7. Prototipo de la máquina de frejol colado Fuente: Elaboración propia.

\section{a. Proceso de mezclado}

Se utiliza un motor eléctrico, dispositivo que transforma la energía eléctrica en energía mecánica por medio de la acción de los campos magnéticos, generados en sus bobinas. Son máquinas eléctricas rotatorias compuestas por un estator y un rotor.

\section{Resultados}

Como resultado de la investigación, se obtuvo una máquina de elaboración automatizada del frejol colado cuyo sabor y textura es igual al que se fabrica de forma tradicional. Funciona de la siguiente manera:

a. Inspección de la máquina, revisión de buenas condiciones y estado de los equipos.

b. La llave termomagnética debe encontrarse apagada (OFF).

c. Conectar la máquina a un tomacorriente de $220 \mathrm{~V}$.

d. Introducir dentro de la olla de acero inoxidable, los ingredientes que se usarán para la elaboración del frejol colado.

e. Encender la llave termomagnética.

f. Iniciar el proceso encendiendo la máquina con el botón de inicio para que se prenda la hornilla. (El sensor óptico reconoce la olla para que se inicie el proceso).

g. Luego de activados, los brazos empiezan a girar lentamente dentro de la olla y cada vez más rápido porque a medida se que vaya cocinando el frejol colado se espese más.

h. A medida que se vaya elaborando el postre, habrá dos sensores, uno de color y otro de temperatura, que configurados con el Controlador Lógico Programable (PLC) harán que se apague la hornilla mediante el control de una electroválvula y dejará de girar el brazo en el punto exacto de cocción. 
i. Por último, se deja reposar.

Dentro de los materiales significativos se utilizó un sensor opto-electrónico, marca SICK de fabricación alemana de 12 a 24 voltios DC empleado para la detección óptica y sin contacto de marcas de contraste. Este equipo no puede estar en contacto con la humedad y suciedad ya que se vería seriamente afectado.

\section{Recomendaciones}

Este postre se debe preparar en una olla gruesa y alta ya que al hervir, la preparación salta y se corre el riesgo de quemarse. Se requiere la utilización de una espátula de madera con mango largo y tapar la olla a fin de evitar algún riesgo.

Debe considerarse la cantidad que se preparará y pesar los ingredientes de manera proporcional. Por ejemplo, para 10 kilos de frejol, hay que agregarle 18 kilos de azúcar y calcular el tiempo de cocción.

El sensor debe colocarse en un lugar específico. Se debe suponer que la temperatura alta puede dańar su uso, ya sea a corto o largo plazo, razón por la cual se tiene que realizar una inspección del mismo.

\section{Conclusiones}

Para el proceso de cocción, se requiere de una cocina automatizada donde se preparará el frejol colado de forma óptima, mediante brazos que sustituirán a los del cocinero. Cuanto más espeso se vuelva el postre, más veloz será el giro del brazo automático, el cual posee un motorreductor, que garantiza movimientos lentos, fuertes y seguros. Manualmente, solo se incorporarán los ingredientes para que la persona encargada no tenga problemas en el uso de la máquina.

Durante la investigación, se halló que en el proceso de elaboración para llegar a un punto óptimo se pueden emplear dos formas: la primera, colocando un sensor de color que determinaría el color ideal y por ende, un mejor producto. La segunda, utilizar una temperatura ideal entre $70^{\circ} \mathrm{C}$ a $80^{\circ} \mathrm{C}$. También se evidenció que la temperatura ideal para obtener un producto de óptima calidad es de $80^{\circ}$. Pero en el caso de utilizar el sensor de color, se tuvo que desestimarlo puesto que los vapores impidieron que se registre el color adecuado del producto final. En consecuencia, esto causó muchos problemas como el no de fijar el sensor en un punto ideal y además, la emanación de vapores malogró el sensor.

Por otro lado, se pudo comprobar que el tiempo de cocción del frejol colado, de una manera convencional o con una máquina de procesamiento no varía. Asimismo, se mantiene el sabor y la contextura del postre.

Finalmente, el conjunto de datos complejos, a partir de sensores de temperatura y al hecho de combinarlos con diferentes datos estadísticos, se torna en herramientas veloces y eficientes para la clasificación, discriminación, reconocimiento e identificación de las muestras. 


\section{Referencias}

Álzate, E. J., Montes, J. W. \& Silva, C. A. (mayo, 2007). Medición de temperatura: sensores termoeléctricos. Scientia et Technica, 1(34).

Amaral, A. F., Ramasamy, A., Castro-Giner, F., Minelli, C., Accordini, S., Sørheim, I. C., ... Jarvis, D. L. (2014). Interaction between gas cooking and GSTM1 null genotype in bronchial responsiveness: results from the European Community Respiratory Health Survey. Thorax, 69(6), 558-564.

Consejo Superior de Investigaciones Científicas (1987). Sensores térmicos. En autor, Introducción a los sensores. Madrid: El Museo Universal.

Hirano, S. B. (2013). Supporting novice cooks through sensor-enhanced computing technologies. 2013 ACM International Joint Conference on Pervasive and Ubiquitous Computing, Zurich, Switzerland.

Hirano, S., Brubaker, J. R., Patterson, D. J. \& Hayes, G. (2013). Detecting cooking state with gas sensors during dry cooking. Recuperado de http://www. jedbrubaker.com/wp-content/uploads/2008/05/ubi1560n-hirano.pdf

Hodgins, D. (1997). The electronic nose: sensor array-based instruments that emulate the human nose. In R. Marsili (Ed.), Techniques for analyzing food aroma (pp. 331-337). New York: Marcel Dekker Inc.
Kalpakjian, S. \& Schmid, S. R. (2002). Manufactura, ingeniería y tecnología. México: Prentice Hall.

Korel, F. \& Balaban, M. (2003). Uses of electronic nose in the food industry. GIDA, 28(5), 505-511.

Quispe, A. \& Calderón, J. (octubre, 2014). Detección de colores por procesamiento digital de imágenes en un equipo de selección de frutos. Ponencia presentada en el IX Congreso virtual VAD.

Smith, K. R. (2006). El uso doméstico de leña en los países en desarrollo y sus repercusiones en la salud. Unasylva, 57, 41-44.

Tudor, M., Markovic, K., Kalit, S., Vahčić, N. \& Kavranek, J. (2014). Application of electronic nose and electronic tongue in the dairy industry. $M l$ jekarstvo, 64(4), 228-244.

Romani, S., Balestra, F., Angioloni, A., Rocculi, P. \& Dalla, M. (December, 2011). Physico-chemical and electronic nose measurements on the study of biscuit baking kinetics. Italian Journal of Food Science, 24(1), 32-40.

Zohora, S. E., Khan, A.M., Srivasava, A. K., \& Hunderwale, N. (November, 2013). Electronic noses application to food analysis using metal oxide sensors: a review. International Journal of Soft Computing and Engineering (IJSCE), 3(5), 199-205. 
